\title{
Attitude and Vibration Control of Flexible Spacecraft Using Singular Perturbation Approach
}

\author{
Morteza Shahravi and Milad Azimi \\ Space Research Institute, Tehran 15875-1774, Iran \\ Correspondence should be addressed to Milad Azimi; azimi@mut.ac.ir
}

Received 4 December 2013; Accepted 16 January 2014; Published 29 May 2014

Academic Editors: Z. Qin, I. Taymaz, and J. Yao

Copyright ( 2014 M. Shahravi and M. Azimi. This is an open access article distributed under the Creative Commons Attribution License, which permits unrestricted use, distribution, and reproduction in any medium, provided the original work is properly cited.

\begin{abstract}
This paper addresses a composite two-time-scale control system for simultaneous three-axis attitude maneuvering and elastic mode stabilization of flexible spacecraft. By choosing an appropriate time coordinates transformation system, the spacecraft dynamics can be divided into double time-scale subsystems using singular perturbation theory (SPT). Attitude and vibration control laws are successively designed by considering a time bandwidths separation between the oscillatory flexible parts motion describing a fast subsystem and rigid body attitude dynamics as a slow subsystem. A nonlinear quaternion feedback control, based on modified sliding mode (MSM), is chosen for attitude control design and a strain rate feedback (SRF) scheme is developed for suppression of vibrational modes. In the attitude control law, the modification to sliding manifold for slow subsystem ensures that the spacecraft follows the shortest possible path to the sliding manifold and highly reduces the switching action. Stability proof of the overall closed-loop system is given via Lyapunov analysis. The proposed design approach is demonstrated to combine excellent performance in the compensation of residual flexible vibrations for the fully nonlinear system under consideration, as well as computational simplicity.
\end{abstract}

\section{Introduction}

In many missions of today's spacecraft with high resolution earth observation payloads and/or large flexible systems, the operation plan requires high precision control capability in order to point at certain area of interest. These missions impose increasingly severe requirements over the modeling and control of spacecraft dynamics. However the flexible structural elements such as solar arrays, antennas, and other light weight parts have received significant focus on providing the control effort for targeting flexible parts such as payloads and tracking maneuver with simultaneous vibration suppression to accomplish mission objectives. Design of such control system poses a challenging problem, including spill-over effects due to the unmodeled dynamics, nonlinear characteristics of rigid-flexible fully coupled dynamics, and unexpected perturbations [1]. From the mathematical point of view, the dynamics of flexible spacecraft involves the coupling of ODEs for attitude dynamics and PDEs for vibration of flexible appendages. This represented by a set of hybrid differential equations (HDE) of motion. Therefore, control strategies have emerged for smoothly shaped maneuvers with vibration excited [2]. Also, the actual performance of controllers is highly sensitive to the error introduced by mathematical model simplification. Therefore the key issues can be classified into modeling error, control/structure interaction, robustness, and so forth [3].

There has been a lot of research and investigation effort for such a problem. Numerical techniques have been reported with analysis and experimental verification. Accordingly, many researchers have surmounted finite dimensional approximation of the original systems. Simultaneous attitude maneuver with vibration suppression has been considered by Vadali [4] and Vadali et al. [5].

The design of robust and practical controllers such as sliding mode control (SMC) which is well known for its powerful robustness and ease of implementation have been presented in some previous studies $[6,7]$. Also in some approaches, 
the spacecraft flexible dynamics are considered as an external perturbation which affected the rigid body motion $[8,9]$.

All the same, in previous works, for the case of threeaxis attitude maneuver with fully nonlinear coupled rigidflexible dynamics, the VSC approach has been modified by ignoring these nonlinear terms. The disadvantage of these modification techniques lies in shifting of the calculated parameters away from what has happened in reality. $\mathrm{Hu}$ proposed a robust nonlinear VSC control theory for 3axis attitude control and vibration suppression of a flexible spacecraft simultaneously with parameter uncertainty and control saturation nonlinearity $[10,11]$. Elsewhere the mentioned researcher $\mathrm{Hu}$ et al. [12] used a control technique which incorporated both SMC and command input shaping for the vibration suppression of a flexible spacecraft in single axis maneuver without proof of global stability of the system. The traditional sliding mode theory with disturbance accommodating control is combined for attitude tracking maneuver of spacecraft [13]. A modified version of classical SMC called smoothing model-reference control is proposed by Lo and Chen in which the attitude tracking performance is increased [14].

The problem of control of residual vibrations has received tremendous interest and poses a challenge task for spacecraft designers. They suggested using smart materials such as shape memory alloys (SMA) and piezoelectric material (PZT), for this problem. The piezoelectric materials have the advantages of high stiffness, light weight, low power consumption, high frequency response, and easy implementation. Using piezoelectric material as actuator (compensator) in the case of surface bounded layers with VSC during attitude maneuver for vibration reduction of flexible appendages in single axis maneuver is proposed by $\mathrm{Hu}$ and $\mathrm{Ma}$ [15]. Azadi et al. [16] studied attitude maneuver control and vibration suppression of a flexible satellite in three-axis rotation using adaptive robust control. The global stability of the fully coupled nonlinear system has not been reported in these researches.

This paper presents a method for degrading the induced vibration and limiting the control action during the slew maneuver based on fully nonlinear dynamic model of the system and using singular perturbation approach. The control of spacecraft for high precision pointing is formulated incorporating control of attitude by modified SMC and SRF techniques simultaneously. Global stability of the complete system has been guaranteed. Numerical simulations show the effectiveness of the proposed controller.

The rest of the paper is organized as follows. Section 2 describes the mathematical modeling of three-axis flexible satellite with embedded piezoelectric materials using physical characteristics of the coupled motion and the singular perturbation theory. The next section states attitude control design based on modified SMC and active vibration suppression based on SRF method using piezoelectric patches. The results of numerical simulations are presented to verify the controller performance in Section 5. Finally concluding remarks are given in Section 6.

\section{Dynamic Modeling}

Figure 1 demonstrates the schematic of the hub with two flexible appendages. A spacecraft model and rigid main body with two clamped loaded Euler-Bernoulli beams bounding with PZT layers are considered to model the elastic deformations of the flexible parts in multiaxis attitude maneuvers. The coordinates used are shown in Figure 1. By choosing the center of the mass of the spacecraft as the body fixed reference frame origin $\left.(O X Y Z)\right|_{b}$, the attitude motions may be decoupled from the translational motions. The beams have the same length $L_{b}$, thickness $t_{b}$, mass per unit length $\rho_{b}$, bending moment of inertia $I_{b}$, and Young's modulus $E_{b}$. The PZT sensors/actuators patches with the length $L_{p}$, thickness $t_{p}$, mass per unit length $\rho_{p}$, bending moment of inertia $I_{p}$, and Young's modulus $E_{p}$ are bounded in both sides of each panel.

The kinematics between the body angular velocity and attitude parameter need to be established. The orientation of the body fixed coordinate $\left.(O X Y Z)\right|_{b}$ with respect to an arbitrary inertial frame $\left.(O X Y Z)\right|_{I}$ may be defined using orthonormal direction cosine $\mathbf{C}(t) \in \mathbf{R}^{3 \times 3}$ matrix [17]:

$$
\left\{\left.(O X Y Z)\right|_{b}(t)\right\}=\mathbf{C}(t)\left\{\left.(O X Y Z)\right|_{I}(t)\right\} .
$$

The unit quaternion, which is a nonminimal representation of an object attitude, completely avoids singular orientations. A quaternion may be presented as a vector $\mathbf{q}=$ $\left[\begin{array}{ll}q_{0} & \mathbf{q}_{1: 3}\end{array}\right] \in R_{4 \times 1}$; that is,

$$
\begin{aligned}
& \mathbf{q}_{1: 3}=\left[\begin{array}{lll}
q_{1} & q_{2} & q_{3}
\end{array}\right]^{T} \\
&=e(t) \operatorname{Sin}\left(\frac{\Phi(t)}{2}\right) \\
& q_{0}=\operatorname{Cos}\left(\frac{\Phi(t)}{2}\right) \\
& \text { with } 0 \leq \Phi(t) \leq 2 \pi,
\end{aligned}
$$

where $\Phi(t)$ is a rotation of a rigid body about the principle Euler rotation axis $e(t)$. The time derivative of the unit quaternion is derived to calculate attitude at any moment:

$$
\dot{\mathbf{q}}(t)=\frac{1}{2}\left[q_{0} \mathbf{I}_{3 \times 3}+{ }^{\times} \mathbf{q}_{1: 3}-\mathbf{q}_{1: 3}^{T}\right]^{T} \boldsymbol{\omega},
$$

where ${ }^{\times} \mathbf{q}_{1: 3}$ is the skew-symmetric matrix of $\mathbf{q}_{1: 3}$ and $\boldsymbol{\omega}=$ $\omega_{x} X_{b}+\omega_{y} Y_{b}+\omega_{z} Z_{b}$ the body angular velocity of the spacecraft.

The displacement $r_{p}(t)$ of any point $p$ on the spacecraft can be defined as

$$
\mathbf{r}(P, t)=\mathbf{r}_{R}^{i}(P) X_{b}+\mathbf{u}^{i}(P, t) Y_{b},
$$

where $\mathbf{r}_{R}^{i}(P)$ is a vector from center of the mass to the undeformed point $p$ and $\mathbf{u}^{i}(P, t), i=1,2$, represents the elastic deflection on $i$ th appendage with respect to nominal position of point $p$.

The velocity of a point $p$ with respect to the body fixed reference frame can be obtained by differentiation of (4):

$$
\mathbf{v}(p, t)=\dot{\mathbf{u}}^{i}(p, t)+\boldsymbol{\omega} \times\left(\mathbf{r}_{R}^{i}(P)+\mathbf{u}^{i}(P, t)\right) .
$$




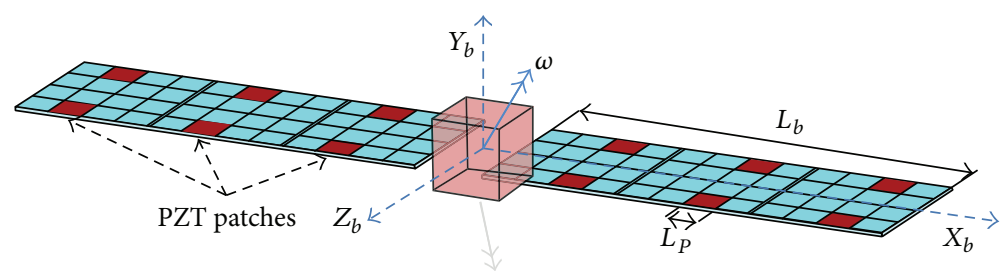

Figure 1: Flexible spacecraft model and parameters.

The kinetic energy of the system including PZT patches can be expressed as

$$
T=\sum_{i=1}^{2} T_{b}^{i}+\sum_{i=1}^{2} \sum_{j=1}^{n_{j}}{ }^{j} T_{p}^{i}
$$

where $T_{b}^{i}, T_{P}^{i}$ and $n_{j}$ represents the kinetic energy of the main structure, the kinetic energy of the $j$ th sensor/actuator pair, and the number of PZT patches, respectively, and can be expressed as

$$
\begin{aligned}
T_{b}^{i}= & \frac{1}{2} \int_{S} \rho_{b} \mathbf{v}(p, t) \cdot \mathbf{v}(p, t) d S=\frac{1}{2} \boldsymbol{\omega}^{T} \mathbf{J}_{b} \boldsymbol{\omega} \\
& +\frac{1}{2} \sum_{i=1}^{2} \int_{a}^{a+L_{b}} \rho_{b}^{i} \dot{\mathbf{u}}^{i}(p, t)^{T} \dot{\mathbf{u}}^{i}(p, t) d x \\
& +\frac{1}{2} \boldsymbol{\omega} \sum_{i=1}^{2} \int_{a}^{a+L_{b}} \rho_{b}^{i}\left({ }^{\times} \mathbf{r}_{R}^{i}(P)+{ }^{\times} \mathbf{u}^{i}(P, t)\right) \dot{\mathbf{u}}^{i}(p, t) d x \\
T_{P}^{i}= & \frac{1}{2} \boldsymbol{\omega}^{T} \mathbf{J}_{p} \boldsymbol{\omega}+\frac{1}{2} \sum_{i=1}^{2} \sum_{j=1}^{n_{j}} \int_{x_{i}}^{x_{i}+L_{P i}}{ }^{j} \rho_{P}^{i} \dot{\mathbf{u}}^{i}(p, t)^{T} \dot{\mathbf{u}}^{i}(p, t) d x \\
& +\frac{1}{2} \boldsymbol{\omega}^{2} \sum_{i=1}^{2} \sum_{j=1}^{n_{j}} \int_{x_{i}}^{x_{i}+L_{P i}}{ }_{j} \rho_{P}^{i}\left({ }^{\times} \mathbf{r}_{R}^{i}(P)+{ }^{\times} \mathbf{u}^{i}(P, t)\right) \\
& \times \dot{\mathbf{u}}^{i}(p, t) d x,
\end{aligned}
$$

where $\mathbf{J}$ is the hub moment of inertia, $a$ is the distance from the hub center to the root of the beam, $L_{b}$ is the length of the flexible beam, $\rho_{b}^{i}$ is the mass per unit length of the $i$ th appendage, ${ }^{j} \rho_{P}^{i}$ is the mass per unit length of the $j$ th PZT patch and $i$ th appendage, $x_{i}$ is starting $x$-coordinate of PZT patch, and $L_{P}$ is the length of the PZT patch.

The potential energy of the flexible structure including PZT patches is considered to be

$$
V=\sum_{i=1}^{2} V_{b}^{i}+\sum_{i=1}^{2} \sum_{j=1}^{n_{j}}{ }^{j} V_{p}^{i}
$$

where $V_{b}^{i}$ and ${ }^{j} V_{P}^{i}$ are the potential energy of the $i$ th main structure and the $j$ th sensor/actuator pair, respectively, and can be written as

$$
\begin{gathered}
V_{b}^{i}=\frac{1}{2} \sum_{i=1}^{2} \int_{a}^{a+L_{b}} E_{b}^{i} I_{b}^{i}\left(\frac{\partial^{2} \mathbf{u}^{i}(P, t)}{\partial x^{2}}\right)^{2} d x \\
{ }^{j} V_{P}^{i}=\frac{1}{2} \sum_{i=1}^{2} \sum_{j=1}^{n_{j}} E_{P}^{i}\left({ }^{j} \omega_{p}^{i}{ }^{j} h_{p}^{i}\right)\left({ }^{j} y^{i^{2}}+{ }^{j} y^{i}{ }^{j} h_{p}^{i}+\frac{{ }^{j} h_{p}^{i 2}}{3}\right) \\
\times \int_{x_{i}}^{x_{i}+L_{P}}\left(\frac{\partial^{2} \mathbf{u}^{i}(p, t)}{\partial x^{2}}\right)^{2} d x,
\end{gathered}
$$

where $E_{b}^{i}$ is the modulus of elasticity for the $i$ th appendage, $I_{b}^{i}$ is the moment of inertia for the beam structure, ${ }^{j} y^{i}$ is the starting point of the PZT as measured from the neutral axis of the beam, ${ }^{j} \omega_{p}^{i}$ is the width of the $j$ th PZT layer and $i$ th appendage, and ${ }^{j} h_{p}^{i}$ is the thickness of each PZT element on $i$ th appendage.

The virtual work done by the external torques $\boldsymbol{\tau}$ and PZT patches is given by

$$
\delta W_{n c}=\sum_{i=1}^{2} \delta W_{\tau}+\sum_{i=1}^{2} \sum_{j=1}^{n_{j}} \delta^{j} W_{p}^{i},
$$

where $\delta^{j} W_{p}^{i}$ is the work done by the $j$ th PZT patch on $i$ th appendage. The work done by the external control torque can be expressed as

$$
\delta W_{\tau}=W_{T} .
$$

The work done by the $j$ th PZT patch is the combination of the conservative and nonconservative work terms defined as an integral over the volume of the PZT patches:

$$
\begin{gathered}
\delta^{j} W_{p}^{i}=\left.\sum_{i=1}^{2} \sum_{j=1}^{n_{j}} \delta^{j} W_{p}^{i}\right|_{c}+\left.\sum_{i=1}^{2} \sum_{j=1}^{n_{j}} \delta^{j} W_{p}^{i}\right|_{n c}, \\
{ }^{j} W_{p}^{i}=\frac{1}{2}{ }^{j} \omega_{p}^{i} \sum_{i=1}^{2} \sum_{j=1}^{n_{j}} \int^{j} x^{j} x^{i}+L_{p} \int^{j} y^{j} y^{i}{ }^{j} h_{p}^{i}\left\{\begin{array}{c}
{ }^{j} E_{3}^{i} \\
{ }^{j} S_{1}^{i}
\end{array}\right\} \\
\times\left[\begin{array}{cc}
1 & 0 \\
0 & -1
\end{array}\right]\left\{\begin{array}{c}
{ }^{j} D_{3}^{i} \\
{ }^{j} T_{1}^{i}
\end{array}\right\} d y d x
\end{gathered}
$$


Using constitutive equation of PZT material (See Appendix A) ${ }^{j} S_{1}^{i}=-y\left(\partial^{2} \mathbf{u}(p, t) / \partial x^{2}\right)$, (13) become

$$
\begin{aligned}
& { }^{j} W_{p}^{i} \\
& =\frac{1}{2}{ }^{j} \varpi_{p}^{i}
\end{aligned}
$$

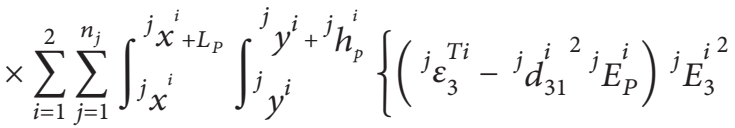

$$
\begin{aligned}
& -\left(2^{j} d_{31}^{i}{ }^{j} E_{P}^{i}{ }^{j} E_{3}^{i}\right. \\
& \left.\left.\times\left(y \frac{\partial^{2} \mathbf{u}^{i}(p, t)}{\partial x^{2}}\right)\right)\right\} d y d x \\
& -\frac{1}{2}{ }^{j} ळ_{p}^{i} \\
& \times \sum_{i=1}^{2} \sum_{j=1}^{n_{j}} \int_{{ }^{j} x_{i}}^{j} x_{i}+L_{P} \int_{j}^{j} y_{i}^{j} y_{i}{ }^{j} h_{p}{ }^{i}\left({ }_{E}^{j} E\left(y \frac{\partial^{2} \mathbf{u}^{i}(p, t)}{\partial x^{2}}\right)^{2}\right) d y d x
\end{aligned}
$$

The last term of (14) is expressed as PZT potential energy ${ }^{j} V_{P}^{i}$; in other words the potential energy expression is composed of conservative works, also introducing

$$
\left.{ }^{j} W_{p}^{i}\right|_{n c}=\frac{1}{2}{ }^{j} \xi_{p}^{i}{ }^{j} \eta_{p}^{i}-\left\{\underline{\mathbf{q}}_{k}^{i}\right\}^{T}\left\{{ }^{j} \mathfrak{R}_{p}^{i}\right\}^{j} \eta_{p}^{i}
$$

with

$$
\begin{aligned}
{ }^{j} \xi_{p}^{i}= & \sum_{i=1}^{2} \sum_{j=1}^{n_{j}} \frac{{ }^{j} \varpi_{p}^{i} L_{P i}}{{ }^{j} h_{p}^{i}}\left({ }^{j} \varepsilon_{3}{ }^{T i}-{ }^{j} d_{31}^{i}{ }^{2}{ }^{i} E_{P}\right) \\
{ }^{j} \eta_{p}^{i}= & \sum_{i=1}^{2} \sum_{j=1}^{n_{j}}{ }^{j} E_{3}^{i} \times{ }^{j} h_{p}^{i} \\
{ }^{j} \mathfrak{R}_{p}^{i}= & \sum_{i=1}^{2} \sum_{j=1}^{n_{j}}{ }^{j} d_{31}^{i}{ }^{j} E_{P}^{i}{ }^{j} \varpi_{p}^{i}\left({ }^{j} y^{i}+\frac{{ }^{j} h_{p}^{i}}{2}\right) \\
& \times \int^{j} x^{j} x^{i}+L_{P i}\left\{\psi^{\prime \prime}(x)\right\}^{T} d x,
\end{aligned}
$$

where $\eta_{p}^{i}$ is the electrode voltage, $\left\{\underline{\mathbf{q}}_{k}^{i}\right\}=\left[q_{1} q_{2} \cdots q_{n}\right]$ is the $k$ th generalized coordinates for $i$ th appendage, and $\{\psi(x)\}$ is the element shape function. Substituting (11) and (15) into (10), the total work can be expressed as

$$
W_{n c}=\frac{1}{2}\{\eta\}^{T}[\Im]\{\eta\}-\{q\}^{T}[R]\{\eta\}+W_{T}
$$

where

$$
\begin{aligned}
{[\mathfrak{I}] } & =\operatorname{diag}\left({ }^{j} \xi_{p}^{i}\right), \\
{[R] } & =\left[\begin{array}{llll}
\left\{{ }^{1} \mathfrak{R}_{p}^{i}\right\} & \left\{{ }^{2} \boldsymbol{R}_{p}^{i}\right\} & \cdots & \left\{{ }^{n_{j}} \boldsymbol{R}_{p}^{i}\right\}
\end{array}\right], \\
\eta & =\left[\begin{array}{llll}
{ }^{1} \eta_{p}^{i} & { }^{2} \eta_{p}^{i} & \ldots & { }^{n} \eta_{p}^{i}
\end{array}\right]^{T} .
\end{aligned}
$$

Utilizing assumed mode method (AMM) and defining

$$
\mathbf{u}_{P}^{i}(x, t)=\sum_{k=1}^{m} \boldsymbol{\psi}_{k}^{T}(x){\underline{\mathbf{q}_{k}^{i}}}_{k}^{i}(t)=\{\boldsymbol{\psi}\}\{\underline{\mathbf{q}}\}
$$

where $\mathbf{u}_{P}^{i}(x, t)$ is elastic displacement of $i$ th appendage which can be discretized using $m$ mode by AMM expansion technique. We substitute (6), (8), and (10) into Lagrange's equations of the motion in terms of quasi-coordinate in a vector form; that is,

$$
\begin{aligned}
& \frac{d}{d t}\left(\frac{\partial L}{\partial \boldsymbol{\omega}}\right)+{ }^{\times} \boldsymbol{\omega}\left(\frac{\partial L}{\partial \boldsymbol{\omega}}\right)=\boldsymbol{\tau}+\mathbf{u} \\
& \frac{d}{d t}\left(\frac{\partial L}{\partial \underline{\dot{\mathbf{q}}}_{k}}\right)-\frac{\partial L}{\partial \underline{\mathbf{q}}_{k}}=\mathbf{0} .
\end{aligned}
$$

Integration over the spatial domains leads to global mass, stiffness and forcing matrixes. In order to account for the structural damping effects in spacecraft dynamics, the Rayleigh's dissipation function may be considered as

$$
T_{d}=\frac{1}{2} \dot{\mathbf{q}}_{k}^{T} \mathbf{C} \dot{\mathbf{q}}_{k} \text {. }
$$

Using (6), (8), (10), and (21) and the extended Hamilton's principle, the attitude dynamic model of a flexible spacecraft can be obtained in the following form:

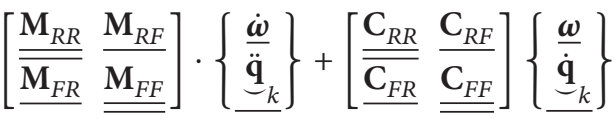

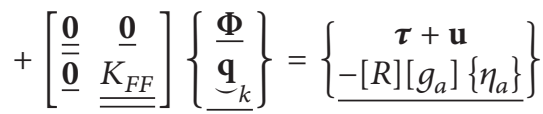

$$
\begin{aligned}
& \left\{\eta_{S}\right\}=\left[g_{s}\right][\mathfrak{\Im}]^{-1}[R]^{T}\left\{\underline{\mathbf{q}}_{k}\right\},
\end{aligned}
$$

where $\left[g_{s}\right]$ and $\left[g_{a}\right]$ represent the sensor and actuator amplifier gains, respectively. The elements of submatrices $\mathbf{M}$, $\mathbf{C}$, and $\mathbf{K}$ are given in Appendix B. The PZT patches will be used as sensors and actuators; accordingly, they will have voltage inputs and outputs. As it can be seen from (22), $[R]$ and $[\mathfrak{\Im}]$ matrices can be decomposed in sensor and actuator parts corresponding to the sensor/actuator voltages, $\left\{\eta_{S}\right\}$ and $\left\{\eta_{a}\right\}$.

\section{Implementation of SPT}

The fundamental idea of this approach is to separate the system dynamics into the slow and fast subsystems. Control 
design may then proceed for each lower-order subsystem, and the results are combined to yield a hybrid stabilized controller for the overall system. For this case, a new variable $\mathbf{Z}$ may be considered as follows:

$$
\begin{aligned}
{\left[\underline{\mathbf{K}_{F F}}\right]\left\{\underline{\mathbf{q}}_{k}\right\} } & =k_{\operatorname{Min}}\left[\underline{\left.\mathbf{K}_{F F}^{R}\right]}\left\{\underline{\mathbf{q}}_{k}\right\}\right. \\
& =\{\mathbf{Z}\} \Longrightarrow\left[\underline{\mathbf{K}_{F F}^{R}}\right]\left\{\underline{\mathbf{q}}_{k}\right\}=\frac{1}{k_{\text {Min }}}\{\mathbf{Z}\}
\end{aligned}
$$

where $k_{\mathrm{Min}}$ is the smallest coefficient of the stiffness matrix $\mathbf{K}_{F F}$. Introducing a new parameter as a singular perturbation parameter, $\varepsilon=\left(1 / k_{\mathrm{Min}}\right)^{0.5}[18]$, (23) become

$$
\left[\underline{\mathbf{K}_{F F}^{R}}\right]\left\{\mathbf{q}_{k}\right\}=\varepsilon^{2}\{\mathbf{Z}\} .
$$

Comparing ${ }^{j} \mathfrak{R}_{p}^{i}$ matrix, with $\sqrt{\mathcal{E}}$, it can be concluded that $O\left({ }^{j} \mathfrak{R}_{p}^{i}\right)=O(\varepsilon)$; therefore, ${ }^{j} \mathfrak{R}_{p}^{i}$ matrix can be written as

$$
{ }^{j} \mathfrak{R}_{p}^{i}=\varepsilon^{j} \mathfrak{R}_{p}^{i R},
$$

where the superscript $R$ denotes to reduced magnitude. Substituting the new variables into the equations of motion, the system becomes

$$
\begin{gathered}
\dot{\boldsymbol{\omega}}=\boldsymbol{\alpha}_{1}^{-1}\left(\boldsymbol{\tau}+\mathbf{u}-\boldsymbol{\alpha}_{2} \boldsymbol{\omega}-\varepsilon^{2} \underline{\underline{\mathbf{K}_{F F}^{R}}} \boldsymbol{\alpha}_{3} \dot{\mathbf{Z}}\right. \\
\left.-\left\{\mathbf{M}_{R F} \mathbf{M}_{F F}^{-1}\left(-\mathbf{Z}-\varepsilon[R]\left[g_{a}\right]\left\{\eta_{a}\right\}\right)\right\}\right), \\
\ddot{\mathbf{Z}}=\varepsilon^{-2} \underline{\underline{\underline{\mathbf{K}_{F F}^{R}}}} \boldsymbol{\gamma}_{1}^{-1}\left(-\varepsilon[R]\left[g_{a}\right]\left\{\eta_{a}\right\}-\boldsymbol{\gamma}_{2} \boldsymbol{\omega}-\varepsilon^{-2}{\underline{\gamma_{3}}}^{\underline{\mathbf{K}_{F F}^{R}-1}} \dot{\mathbf{Z}}\right. \\
\left.-\mathbf{M}_{F R} \mathbf{M}_{R R}^{-1}(\boldsymbol{\tau}+\mathbf{u})-\mathbf{Z}\right) .
\end{gathered}
$$

The slow subsystem can be obtained by setting terms of $O\left(\varepsilon^{2}\right)$ and higher equal to zero in the equations of motion:

$$
\mathbf{Z}_{\text {Slow }}=\left(-\mathbf{M}_{F R} \mathbf{M}_{R R}^{-1}(\boldsymbol{\tau}+\mathbf{u})-\varepsilon[R]\left[g_{a}\right]\left\{\eta_{a}\right\}-\boldsymbol{\alpha}_{2} \boldsymbol{\omega}\right) .
$$

Substituting (28) in (26) yields:

$$
\begin{aligned}
\dot{\boldsymbol{\omega}}_{\text {Slow }}=\boldsymbol{\alpha}_{1}^{-1}(\boldsymbol{\tau} & -\boldsymbol{\alpha}_{2} \boldsymbol{\omega} \\
& \left.-\left.\mathbf{M}_{R R} \mathbf{M}_{F F}^{-1}\left(-\gamma_{2} \boldsymbol{\omega}-\mathbf{M}_{F R} \mathbf{M}_{R R}^{-1}(\boldsymbol{\tau}+\mathbf{u})\right)\right|_{\text {Slow }}\right)
\end{aligned}
$$

The term including control voltage of PZT actuators is of $O(\varepsilon)$ and consequently can be ignored since its magnitude is much less than the other terms, which leads to an $O(\varepsilon)$ approximation of the slow subsystem.

Fast subsystem can be obtained by setting $\psi_{f}=t / \varepsilon$, introducing $\mathbf{Z}_{\text {Fast }}=\mathbf{Z}-\mathbf{Z}_{\text {Slow }}$, lead the slow variables as constant in fast time scale [19] and considering the terms of $O\left(\varepsilon^{2}\right)$ and higher equal to zero so the equation of the motion for fast subsystem can be expressed as

$$
\begin{aligned}
\frac{d^{2}}{d \psi_{f}^{2}} \mathbf{Z}_{\text {Fast }}=\mathbf{K}_{F F}^{R} \gamma_{1}^{-1}(- & \left.\left(\varepsilon[R]\left[g_{a}\right]\left\{\eta_{a}\right\}\right)\right|_{\text {Fast }}-\gamma_{3} \frac{d}{d \psi_{f}} \mathbf{Z}_{\text {Fast }} \\
& \left.-\mathbf{Z}_{\text {Fast }}-\left.\mathbf{M}_{F R} \mathbf{M}_{R R}^{-1}(\boldsymbol{\tau}+\mathbf{u})\right|_{\text {Fast }}\right) .
\end{aligned}
$$

The equation of the motion of (26) becomes

$$
\begin{aligned}
& {\left[\begin{array}{cc}
\underline{\underline{\boldsymbol{\alpha}}} & \underline{\mathbf{0}} \\
\underline{\overline{\mathbf{0}}} & \underline{\underline{\gamma_{1}}}
\end{array}\right] \cdot\left\{\begin{array}{c}
\underline{\dot{\boldsymbol{\omega}}} \\
\underline{\ddot{\psi}}_{f}
\end{array}\right\}+\left[\begin{array}{cc}
\underline{\underline{\boldsymbol{\alpha}_{2}+\mathbf{M}_{R F} \mathbf{M}_{F F} \boldsymbol{\gamma}_{2}}} & \underline{\mathbf{0}} \\
\underline{\mathbf{0}} & \underline{\underline{\gamma_{3}}}
\end{array}\right]} \\
& \left\{\begin{array}{c}
\underline{\underline{\omega}} \\
\dot{\boldsymbol{\psi}}_{f}
\end{array}\right\}+\left[\begin{array}{cc}
\underline{\underline{\mathbf{0}}} & \underline{\mathbf{0}} \\
\underline{\mathbf{0}} & \underline{\underline{\boldsymbol{\kappa}_{F F}}}
\end{array}\right] \cdot\left\{\begin{array}{c}
\underline{\underline{\Phi}} \\
\underline{\psi_{f}}
\end{array}\right\} \\
& =\left\{\begin{array}{c}
\mathbf{M}_{R R}^{-1} \boldsymbol{\alpha}_{1}(\boldsymbol{\tau}+\mathbf{u}) \\
-\varepsilon[R]\left[g_{a}\right]\left\{\eta_{a}\right\}-\mathbf{M}_{F R} \mathbf{M}_{R R}^{-1}(\boldsymbol{\tau}+\mathbf{u})
\end{array}\right\},
\end{aligned}
$$

where $\alpha_{i}, \gamma_{i}(i=1,2,3)$ are defined as

$$
\begin{aligned}
& \boldsymbol{\alpha}_{1}=\mathbf{M}_{R R}-\mathbf{M}_{R F} \mathbf{M}_{F F}^{-1} \mathbf{M}_{F R}, \\
& \boldsymbol{\alpha}_{2}=\mathbf{C}_{R R}-\mathbf{M}_{R F} \mathbf{M}_{F F}^{-1} \mathbf{C}_{F R}, \\
& \boldsymbol{\alpha}_{3}=\mathbf{C}_{R F}-\mathbf{M}_{R F} \mathbf{M}_{F F}^{-1} \mathbf{C}_{F F}, \\
& \boldsymbol{\gamma}_{1}=\mathbf{M}_{F F}-\mathbf{M}_{F R} \mathbf{M}_{R R}^{-1} \mathbf{M}_{R F}, \\
& \boldsymbol{\gamma}_{2}=\mathbf{C}_{F R}-\mathbf{M}_{F R} \mathbf{M}_{R R}^{-1} \mathbf{C}_{R R}, \\
& \boldsymbol{\gamma}_{3}=\mathbf{C}_{F F}-\mathbf{M}_{F R} \mathbf{M}_{R R}^{-1} \mathbf{C}_{R F},
\end{aligned}
$$

\section{Controller Design}

In the present work, three-axis attitude maneuver and vibration control are considered.

The quaternions are chosen for representation of the attitude of the spacecraft. By taking angular velocity and quaternion vectors, a modified sliding manifold is being proposed as

$$
\mathbf{S}=\boldsymbol{\omega}_{e}+\mathbf{K} \tanh \left(q_{0-e}\right) \mathbf{q}_{1: 3-e},
$$

where $\boldsymbol{\omega}_{e}=\boldsymbol{\omega}-\boldsymbol{\omega}_{d}$ is the spacecraft angular velocity tracking error, $\mathbf{q}_{e}=\mathbf{q}\langle\times\rangle \mathbf{q}_{d}^{-1}$ is the quaternion tracking error, in which $\langle\times\rangle$ is the quaternion products, $\mathbf{q}_{d}$ and $\boldsymbol{\omega}_{d}$ are the desired quaternion and angular velocity, respectively.

Theorem 1. The control objective is to stabilize the flexible spacecraft by forcing the rigid body modes to follow some desired trajectories, while simultaneously reducing the elastic modes. The desired attitude maneuver with high mode flexibility can be realized, if the sliding condition $\dot{V} \prec 0$ is satisfied. 
Proof. The desired state that slides on the sliding surface can be shown to be asymptotically stable by choosing the candidate Lyapunov function as

$$
V=\frac{1}{2} \mathbf{S}^{T} \boldsymbol{\alpha}_{1} \mathbf{S}
$$

The proposed Lyapunov function is valid since it vanishes at equilibrium point $\mathbf{S}=\mathbf{0}$ and is globally positive definite for $\mathbf{S} \neq \mathbf{0}$ since $\boldsymbol{\alpha}_{1}$ is positive definite. The time derivative of Lyapunov function is given by

$$
\dot{V}=\frac{1}{2} \mathbf{S}^{T} \boldsymbol{\alpha}_{1} \dot{\mathbf{S}}
$$

where the derivative of sliding surface is defined as

$$
\dot{\mathbf{S}}=\dot{\boldsymbol{\omega}}+\mathbf{K} \tanh \left(q_{0}\right) \dot{\mathbf{q}}_{1: 3}
$$

From the equation of the motion we have

$$
\begin{aligned}
\boldsymbol{\alpha}_{1} \dot{\boldsymbol{\omega}}= & -\left\{\boldsymbol{\alpha}_{2}+\mathbf{M}_{R F} \mathbf{M}_{F F}^{-1} \boldsymbol{\gamma}_{2}\right\} \boldsymbol{\omega} \\
& +\left\{1-\mathbf{M}_{R F} \mathbf{M}_{F F}^{-1} \mathbf{M}_{F R} \mathbf{M}_{R R}^{-1}\right\}(\mathbf{u}+\boldsymbol{\tau}),
\end{aligned}
$$

where $\mathbf{u}$ is the control torque generated by actuators placed on rigid main body. Multiply each side of (36) by $\boldsymbol{\alpha}_{1}$ combined with (37) leads to the following expression for $\dot{V}$ :

$$
\begin{aligned}
\dot{V}=\mathbf{S}^{T}( & -\left\{\boldsymbol{\alpha}_{2}+\mathbf{M}_{R F} \mathbf{M}_{F F}^{-1} \boldsymbol{\gamma}_{2}\right\} \boldsymbol{\omega} \\
& \left.+\left\{\mathbf{M}_{R R}^{-1} \boldsymbol{\alpha}_{1}\right\}(\mathbf{u}+\boldsymbol{\tau})+\left(K_{1} \tanh \left(q_{0}\right) \boldsymbol{\alpha}_{1} \dot{\mathbf{q}}_{1: 3}\right)\right),
\end{aligned}
$$

which is clearly negative definite provided that $K_{1}>0$. The controller designed by variable structure approach consists of two different tasks. First one is to define an appropriate sliding surface and the other one is to improve the sliding condition, which it commands the states remain on the sliding surface. By solving the above equation for the control input, the external control torque can be derived in such a way that

$$
\mathbf{u}=\mathbf{u}_{\mathrm{eq}}+\mathbf{u}_{\mathrm{VS}}
$$

where the variable structure and equivalent parts of controller input are defined as

$$
\begin{gathered}
\mathbf{u}_{\mathrm{VS}}=-\left(K_{2} \mathbf{S}(t)\right)-\left(K_{3} \tanh \left(\frac{\mathbf{S}(t)}{P^{2}}\right)\right) \\
\mathbf{u}_{\mathrm{eq}}=\left(\mathbf{M}_{R R}^{-1} \boldsymbol{\alpha}_{1}\right)^{-1}\left(\left\{\boldsymbol{\alpha}_{2}+\mathbf{M}_{R F} \mathbf{M}_{F F}^{-1} \boldsymbol{\gamma}_{2}\right\} \boldsymbol{\omega}\right. \\
\left.-\left\{\mathbf{M}_{R R}^{-1} \boldsymbol{\alpha}_{1} \boldsymbol{\tau}\right\}-\left(K_{1} \tanh \left(q_{0}\right) \boldsymbol{\alpha}_{1} \dot{\mathbf{q}}_{1: 3}\right)\right) .
\end{gathered}
$$

The equivalent control $\mathbf{u}_{\mathrm{eq}}$ part turns the sliding surface $\mathbf{S}(t)$ into an invariant manifold for the system, to ensure that $\dot{\mathbf{S}}=0$. Whereas the variable structure part $\mathbf{u}_{\mathrm{VS}}$ is chosen to ensure that the $\mathbf{S}=0$; thus, the designing surface is attractive and the desired condition can be reached in finite time.
Substitutions of (40) and (41) into the sliding condition yield

$$
\dot{V}=-\mathbf{S}^{T}(t)\left\{\left(K_{2} \mathbf{S}(t)\right)+\left(K_{3} \tanh \left(\frac{\mathbf{S}(t)}{P^{2}}\right)\right)\right\} \prec 0
$$

where $K_{n}$ with $n=1,2,3$ are positive definite matrices, and $P^{2}$ is a scalar sharpness function that regulates the control action rates. Note that the term $\dot{\mathbf{q}}_{1: 3}$ in (41) introduces nonlinear terms in variable-structure controller. This implies from theorem and the $K_{n}$ values that as $t \longrightarrow \infty$ the control objective $\left[\begin{array}{ll}\mathbf{q} & \boldsymbol{\omega}\end{array}\right]_{d}^{T}=\left[\begin{array}{lll}1 & \mathbf{0}_{1 \times 3} & \mathbf{0}_{1 \times 3}\end{array}\right]^{T}$ and the asymptotic global stability can be achieved due to the $\dot{V}=-(1 / 2) \mathbf{S}^{T} \mathbf{u}_{\mathrm{VS}}$.

It can be seen from (40) and (41) that the stability and robustness of controller performance are guaranteed if the upper bounds of the perturbations are known. This knowledge may cause the controller to produce the overconservative high gain $K_{3}$. This may cause chattering phenomenon. In order to overcome this source of degradation or overaction, the hyperbolic tangent function is used to reduce chattering.

Also, the active vibration suppression system using the PZT sensor and actuator can actively suppress the solar and environmental induced vibration to the flexible appendages during attitude maneuver. Since no external field is applied to the sensor layer, the electric displacement developed on the sensor surface is directly proportional to the strain acting on it. Also PZT materials can be used as strain rate sensors. The output current of the PZT sensor measures the moment rate of the flexible appendages. This current is converted into the open circuit sensor voltage $V_{S}$ using a signal conditioning device with the gain $G_{C}$ and applied to an actuator with a suitable controller gain. Thus, the sensor output voltage is obtained as

$$
\begin{aligned}
V_{S}(t) & =G_{C} i(t) \\
& =G_{C} e_{31}\left(\frac{h_{b}}{2}+h_{p}\right) \omega_{p} \int_{0}^{L_{P}} \frac{\partial^{2}}{\partial x^{2}} \psi_{k}(x) \dot{\mathbf{q}}_{k}(t) d x,
\end{aligned}
$$

where $i(t)$ is circuit current, and the indices $b$ and $p$ explain the beam and PZT structures, respectively. This sensor voltage is given as input to the controller and the output of the controller is the controller gain multiplied by the sensor voltage. Thus, the input voltage to the actuator $V_{a}$, in other words the controller input $u(t)$, is given by

$$
V_{a}(t)=u(t)=\mathbf{K}_{p} \times V_{S}(t)
$$

where $\mathbf{K}_{p}$ is the controller gain matrix. Note that feedback gain matrix $\mathbf{K}_{p}$ consists of each feedback gain which is associated with each flexible PZT patch. The actuator equation is derived from the converse PZT equation and the relative control force $\mathbf{f}_{\text {ctrl }}$ produced by the actuator that is applied on the appendages is obtained using bending moment theory:

$$
\mathbf{f}_{\mathrm{ctrl}}=E_{P} d_{31} \omega_{p}\left(\frac{h_{P}+h_{b}}{2}\right) \int_{0}^{L_{P}} \frac{\partial}{\partial x} \psi(x) d x V_{a}(t)
$$


TABLE 1: Parameters of flexible spacecraft.

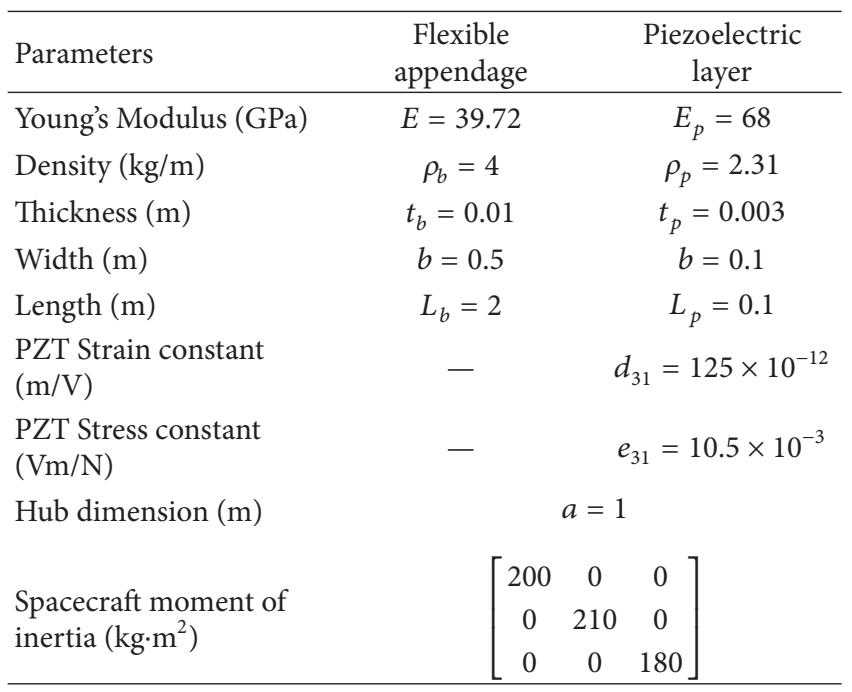

\section{Numerical Simulations and Results}

Simulation of fully nonlinear 3-axis attitude maneuver of a flexible spacecraft has been carried out using MATLAB/SIMULINK software to demonstrate the performance of proposed approach. The proposed control system objective of a flexible spacecraft model is to reduce the induced vibration and tracking a target in sample mission.

The desired maneuver is $160^{\circ}$ slew with simultaneous vibration suppression. This is usually a fast and large angle maneuver. The numerical values of the parameters used in the simulation study are presented in Table 1.

The initial conditions for the angular velocity are set to $\boldsymbol{\omega}\left(t_{0}\right)=\left[\begin{array}{lll}0 & 0 & 0\end{array}\right]^{T}$ and for quaternion parameters are given by $\mathbf{q}\left(t_{0}\right)=\left[\begin{array}{llll}0.174 & -0.263 & 0.789 & -0.526\end{array}\right]^{T}$. The first two flexible modes are retained in the model for discretization of elastic deformations. For control implementation, design parameters are considered as $\mathbf{K}_{1}=0.4 \mathbf{I}_{3 \times 3}, \mathbf{K}_{2}=15 \mathbf{I}_{3 \times 3}$ and $\mathbf{K}_{3}=0.28 \mathbf{I}_{3 \times 3}$.

Dynamical behavior of the controlled system is shown in Figures 2, 3, 4, 5, 6, 7, 8, and 9. Smoothness and convergence of attitude error in terms of quaternions and angular rate are shown in Figures 2 and 3. Figures 4-6 show the required control torques for different states.

As shown in these figures, using SPT and accounting complete coupling of flexible/rigid dynamics in controller design process causes actually better response of the closed loop system and controller performance. Also, active suppression of structural vibration causes smooth and fine actuation of attitude controller. This is an important characteristic for actual implementation of the controller. As shown in Figure 6, using classical SMC (without modification) causes steady state error arising from flexible modes excitation. Convergence of the flexible body coordinates and PZT actuation voltage are shown in Figures 7-9.

\section{Conclusion}

A new methodology and control design approach for multiaxis attitude maneuver and vibration suppression of flexible spacecraft has been proposed. The proposed scheme is based on mapping of the fully coupled nonlinear system dynamics into slow and fast subsystem domain using SPT and designing of hybrid control modified SMC/SRF for system. The hybrid controller can obtain asymptotical reference attitude and suppress structural vibrations excited by, for example, rapid maneuvers or other disturbances. Stability proof of the overall system has been proved using Lyapunov stability analysis.

It has been shown that the performance of the resultant closed-loop system being improved compared to those of traditional, while fast targeting, suppression of residual structural vibration and assuring overall stability.

\section{Appendices}

\section{A. Piezoelectric Constitutive Equations}

The $3 \mathrm{D}$ constitutive equation for a piezoelectric element can be shown to have the following standard notation [20] as

$$
\left[\begin{array}{l}
\frac{D_{i}}{S_{j}}
\end{array}\right]=\left[\begin{array}{lll}
\frac{\varepsilon_{i}^{T}}{d_{i j}^{1 T}} & \frac{d_{i j}^{1}}{S_{i j}^{E 1}} & \underline{d_{i j}^{2}} \\
\frac{\underline{0}}{d_{i j}^{2}} & \underline{0} & \underline{S_{i j}^{E 2}}
\end{array}\right]\left[\underline{E_{i}} \frac{\underline{T_{j}}}{\underline{y}}\right],
$$

where $D_{i}(i=1,2,3)$ denotes the electric displacement along the $i$ th axis, $E_{i}(i=1,2,3)$ represents the applied electrical field density, $S_{i}(i=1, \ldots, 6)$ represents strain, $\sigma_{i}(i=$ $1, \ldots, 6)$ represents the stress, $\underline{\varepsilon}_{i}^{T}(i=1,2,3), S_{i j}^{E k}(i=$ $1,5, j=1,2,3,5, k=1,2)$, and $d_{i j}^{k}(i=1, \overline{1,3} j=$ $1,3,5, k=1,2)$ are permittivity, elastic compliance, and piezoelectricity (strain) coefficient constants of the PZT material, respectively:

$$
\begin{gathered}
\underline{\varepsilon_{i}^{T}}=\left[\begin{array}{ccc}
\varepsilon_{1}^{T} & 0 & 0 \\
0 & \varepsilon_{1}^{T} & 0 \\
0 & 0 & \varepsilon_{3}^{T}
\end{array}\right], \quad \underline{d_{i j}^{1}}=\left[\begin{array}{ccc}
0 & 0 & 0 \\
0 & 0 & 0 \\
d_{31} & d_{31} & d_{33}
\end{array}\right], \\
\underline{d i j}_{i j}^{2}=\left[\begin{array}{ccc}
0 & d_{15} & 0 \\
d_{15} & 0 & 0 \\
0 & 0 & 0
\end{array}\right] \\
\underline{S_{i j}^{E 1}}=\left[\begin{array}{lll}
S_{11}^{E} & S_{12}^{E} & S_{13}^{E} \\
S_{12}^{E} & S_{11}^{E} & S_{13}^{E} \\
S_{13}^{E} & S_{13}^{E} & S_{13}^{E}
\end{array}\right], \quad \underline{S_{i j}^{E 2}}=\left[\begin{array}{ccc}
S_{55}^{E} & 0 & 0 \\
0 & S_{55}^{E} & 0 \\
0 & 0 & S_{55}^{E}
\end{array}\right]
\end{gathered}
$$

The strain condition based on Euler-Bernoulli beam theory is defined as

$$
\varepsilon_{x}=-y \frac{\partial^{2} w}{\partial x^{2}}, \quad \varepsilon_{y}=\varepsilon_{z}=\gamma_{x y}=\gamma=\gamma_{y x}=0 .
$$




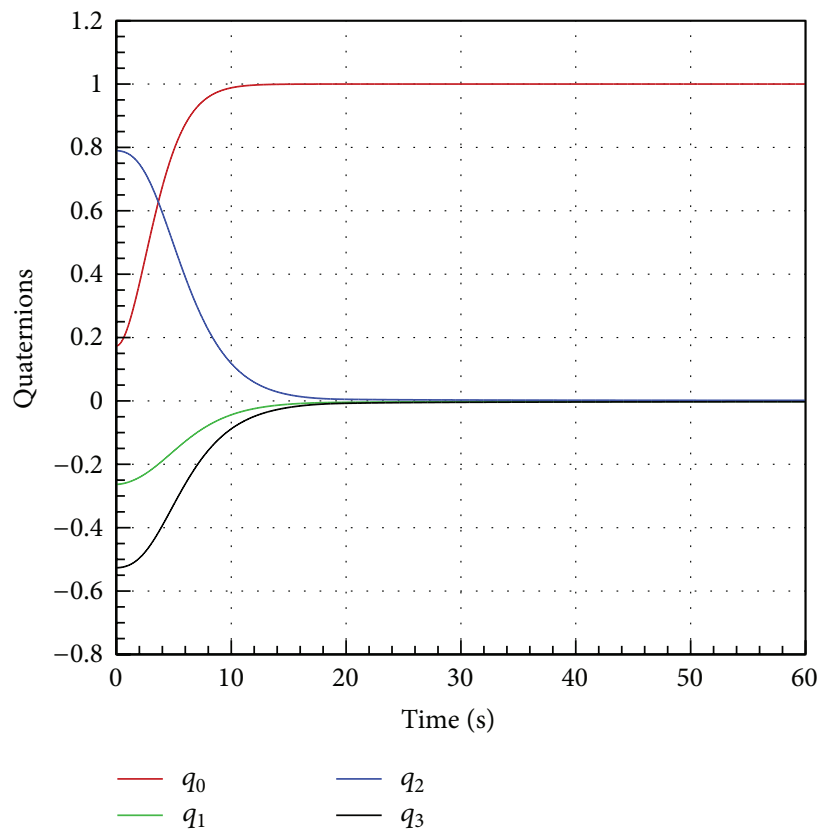

Figure 2: Time history of Attitude quaternion.

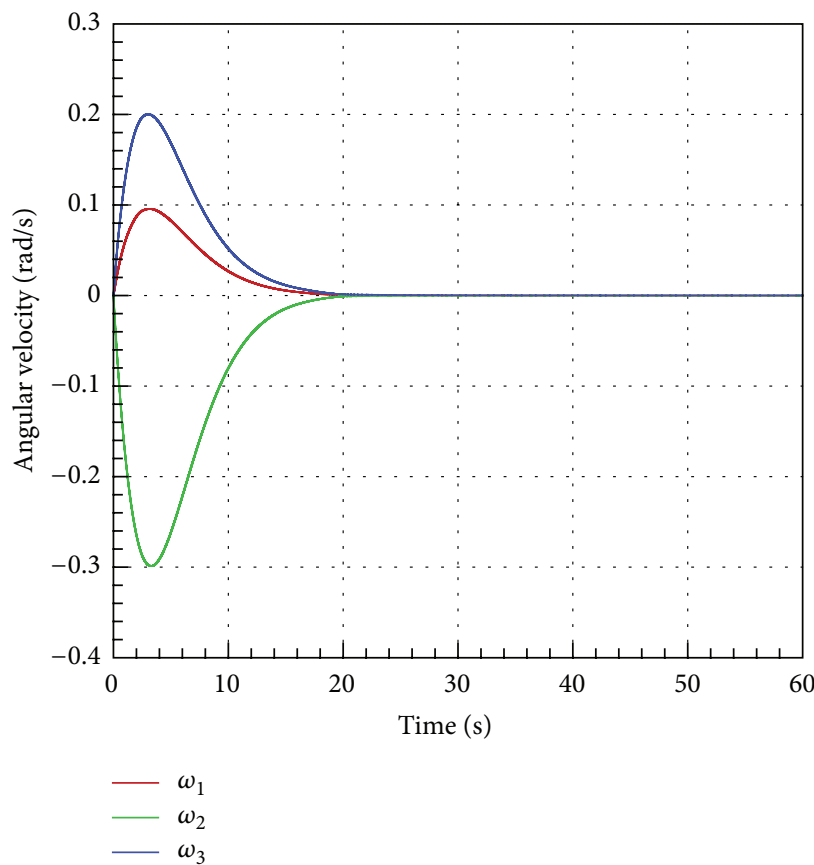

FIgURE 3: Time history of angular velocity.

It can be seen from (A.4) that (A.1) reduced to 1-D Constitutive equation of PZT material and is thereby found to be

$$
\left\{\begin{array}{c}
D_{3} \\
S_{1}
\end{array}\right\}=\left[\begin{array}{ll}
\varepsilon_{3}^{T} & d_{31} \\
d_{31} & S_{11}^{E}
\end{array}\right]\left\{\begin{array}{c}
E_{3} \\
T_{1}
\end{array}\right\}
$$

Using the fact that $S_{i j}^{E}=E_{P}^{-1}$, where $E_{P}$ is Young's modulus, (A.3) can be expressed as below: 

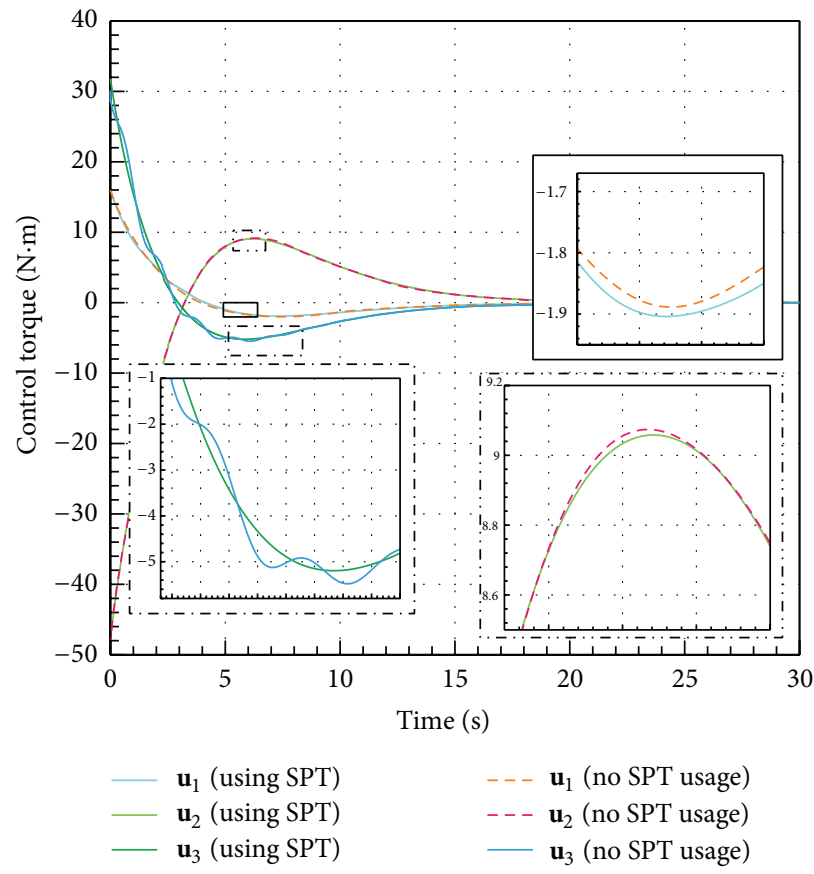

FIGURE 4: Time history of control torque with active vibration suppression.

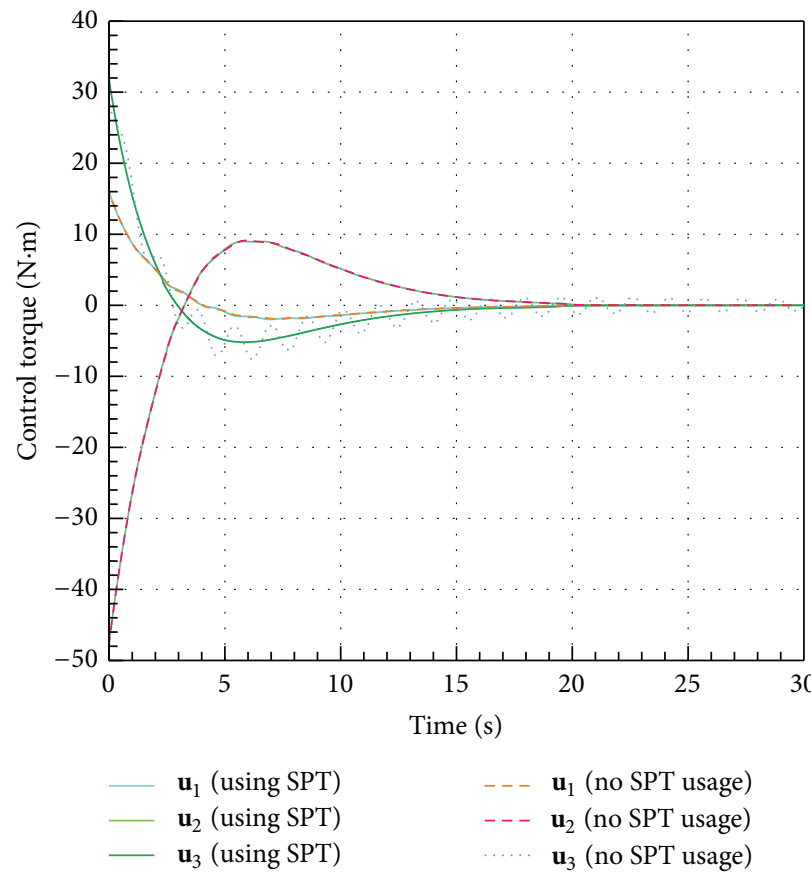

FIGURE 5: Time history of control torque without active vibration suppression.

\section{B. The Elements of the Matrices M, C, K and J}

The elements of the sub matrices of the system are:

$$
+\sum_{i=1}^{2} \sum_{j=1}^{n_{j}} \int_{x_{i}}^{x_{i}+L_{P i}}{ }^{j} \rho_{P}^{i}{ }^{i} \mathbf{u}^{2} d x
$$

$$
\begin{array}{r}
\mathbf{J}=\mathbf{J}_{h}+\mathbf{J}_{b}+\mathbf{J}_{p}=\left[J^{i j}\right]_{3 \times 3}, \\
J^{11}=I_{X X}+\sum_{i=1}^{2} \int_{a}^{a+L_{b}} \rho_{b}^{i{ }^{i} \mathbf{u}^{2} d x}
\end{array}
$$$$
J^{12}=J^{21}=-\sum_{i=1}^{2} \int_{a}^{a+L_{b}} \rho_{b}^{i i} \mathbf{u} d x
$$$$
+\sum_{i=1}^{2} \sum_{j=1}^{n_{j}} \int_{x_{i}}^{x_{i}+L_{P i}} \rho_{P}^{i}{ }^{i} \mathbf{u} d x
$$ 


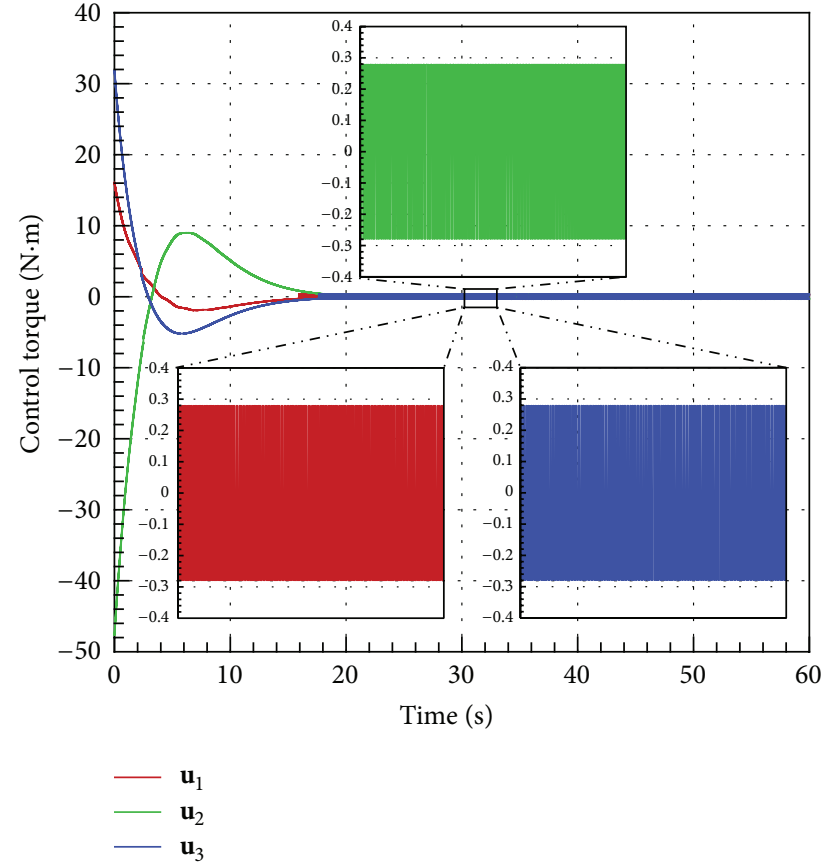

Figure 6: Time history of control action using classical (Notmodified) SMC.

$$
\begin{aligned}
& J^{13}=J^{31}=0, \\
& J^{22}=I_{Y Y}, \\
& J^{23}=J^{32}=0, \\
& J^{33}=I_{Z Z}+\sum_{i=1}^{2} \int_{a}^{a+L_{b}} \rho_{b}^{i} \mathbf{u}^{2} d x \\
& +\sum_{i=1}^{2} \sum_{j=1}^{N_{j}} \int_{x_{i}}^{x_{i}+L_{P i}} \rho_{P}^{i}{ }^{i} \mathbf{u}^{2} d x \\
& \mathbf{M}_{R R}=\left[M_{R R}^{i j}\right]_{3 \times 3}, \\
& M_{R R}^{11}=I_{X X}+\sum_{i=1}^{2}\left\{{ }^{i} \underline{\mathbf{q}}_{k}^{T}{ }^{i} \Upsilon_{y y}{ }^{i} \underline{\mathbf{q}}_{k}+{ }^{i} a_{y}\left(\rho_{b}+\sum_{j=1}^{N_{j}}{ }^{j} \rho_{P}\right)\right. \\
& \left.+2^{i} a_{y}{ }^{i} \Upsilon_{y}^{T}{ }^{i} \underline{q}_{k}\right\} \\
& M_{R R}^{12}=M_{R R}^{21}=-\sum_{i=1}^{2}\left\{{ }^{i} \Upsilon_{y x}^{T} \underline{-}_{k} \mathbf{q}^{i} a_{x}{ }^{i} a_{y}+{ }^{i} a_{x}{ }^{i} \Upsilon_{y y}^{T}{ }^{i} \mathbf{q}_{k}\right. \\
& \left.+{ }^{i} a_{y}\left(\rho_{b} \frac{L_{b}^{2}}{2}+\sum_{j=1}^{N_{j}} \rho_{P} \frac{{ }^{j} L_{P}^{2}}{2}\right)\right\}, \\
& M_{R R}^{13}=M_{R R}^{31}=0,
\end{aligned}
$$

$$
\begin{aligned}
& M_{R R}^{22}=I_{Y Y}, \\
& M_{\mathrm{RR}}^{23}=M_{\mathrm{RR}}^{32}=0, \\
& M_{R R}^{33}=I_{Z Z}+\sum_{i=1}^{2}\left\{{ }^{i} \underline{\mathbf{q}}_{k}^{T}{ }^{i} \Upsilon_{y y}{ }^{i} \underline{\mathbf{q}}_{k}+{ }^{i} a_{y}^{2}\left(\rho_{b}+\sum_{j=1}^{N_{j}} \rho_{p}\right)\right\} \\
& +2^{i} a_{y}{ }^{i} \Upsilon_{y}^{T}{ }^{i} \underline{q}_{k}+\rho_{b} \int_{0}^{L_{b i}} x^{2} d x \\
& +\sum_{j=1}^{N_{j}} \rho_{P} \int_{x_{i}}^{x_{i}+L_{P i}} x^{2} d x \\
& \mathbf{M}_{R F}=\left[\begin{array}{ll}
{ }^{1} a_{x} & 2 \\
& { }^{2} a_{x}
\end{array}\right]\left[\begin{array}{cc}
{ }^{1} \Upsilon_{y}^{T} & \mathbf{0} \\
\mathbf{0} & { }^{2} \Upsilon_{y}^{T}
\end{array}\right]-\left[\begin{array}{cc}
{ }^{1} \Upsilon_{y x}^{T} & { }^{2} \Upsilon_{y x}^{T}
\end{array}\right], \\
& \mathbf{M}_{F R}=\left[\begin{array}{ll}
{ }^{1} a_{x} & 2 \\
& { }^{2} a_{x}
\end{array}\right]\left[\begin{array}{cc}
{ }^{1} \Upsilon_{y}^{T} & \mathbf{0} \\
\mathbf{0} & { }^{2} \Upsilon_{y}^{T}
\end{array}\right]-\left[\begin{array}{cc}
{ }^{1} \Upsilon_{y x}^{T} & { }^{2} \Upsilon_{y x}^{T}
\end{array}\right] \text {, } \\
& \mathbf{M}_{F F}=\left[\begin{array}{cc}
{ }^{1} \Upsilon_{y y} & \mathbf{0} \\
\mathbf{0} & { }^{2} \Upsilon_{y y}
\end{array}\right] \\
& \mathbf{C}_{R R}=\left[C_{R}^{i j}\right]_{3 \times 3}, \\
& C_{R R}^{11}=\omega_{z} \sum_{i=1}^{2}\left\{{ }^{i} \Upsilon_{y x}^{T}{ }^{i} \underline{q}_{k}+{ }^{i} a_{x}{ }^{i} a_{y}+{ }^{i} a_{x}{ }^{i} \Upsilon_{y}^{T}{ }^{i} \underline{q}_{k}\right. \\
& \left.+{ }^{i} a_{y}\left(\rho_{b} \frac{L_{b}^{2}}{2}+\sum_{j=1}^{N_{j}}{ }^{j} \rho_{P} \frac{{ }^{j} L_{P}^{2}}{2}\right)\right\} \\
& +2 \sum_{i=1}^{2}\{\underbrace{i} \dot{\dot{\mathbf{q}}}_{k}^{T}{ }^{i} \Upsilon_{y y}^{T}{ }^{i} \underline{G}_{k}+{ }^{i} a_{y}{ }^{i} \Upsilon_{y}^{T}{ }^{i} \underline{\dot{\mathbf{q}}}_{k}\}, \\
& C_{R R}^{21}=\omega_{z}\left\{\sum _ { i = 1 } ^ { 2 } \left\{{ }^{i} \underline{\mathbf{q}}_{k}^{T}{ }^{i} \Upsilon_{y y}^{T}{ }^{i} \underline{q}_{k}+{ }^{i} a_{y}^{2}\left(\rho_{b}+\sum_{j=1}^{N_{j}} \rho_{P}\right)\right.\right. \\
& \left.+2 a_{y}\left(\rho_{b}+\sum_{j=1}^{N_{j}} \rho_{P}\right){ }^{i} \Upsilon_{y}^{T}{ }^{i} \underline{q}_{k}\right\} \\
& \left.+\left(I_{Z Z}-I_{y y}\right)\right\} \\
& C_{R R}^{31}=0, \\
& C_{R R}^{21}=-2 \sum_{i=1}^{2}\left\{{ }^{i} \Upsilon_{y x}^{T}{ }^{i} \underline{\underline{\mathbf{q}}}_{k}+{ }^{i} a_{x}{ }^{i} \Upsilon_{y}^{T}{ }^{i} \underline{\dot{\mathbf{q}}}_{k}\right\}+\omega_{z}\left(I_{X X}-I_{y y}\right), \\
& C_{R R}^{22}=\omega_{z} \sum_{i=1}^{2}\left\{{ }^{i} \Upsilon_{y x}^{T}{ }^{i} \underline{\mathbf{q}}_{k}+{ }^{i} a_{x}{ }^{i} a_{y}+{ }^{i} a_{x}{ }^{i} \Upsilon_{y}^{T}{ }^{i} \underline{\mathbf{q}}_{k}\right. \\
& \left.+{ }^{i} a_{y}\left(\rho_{b} \frac{L_{b}^{2}}{2}+\sum_{j=1}^{N_{j}}{ }^{j} \rho_{P} \frac{{ }^{j} L_{P}^{2}}{2}\right)\right\},
\end{aligned}
$$




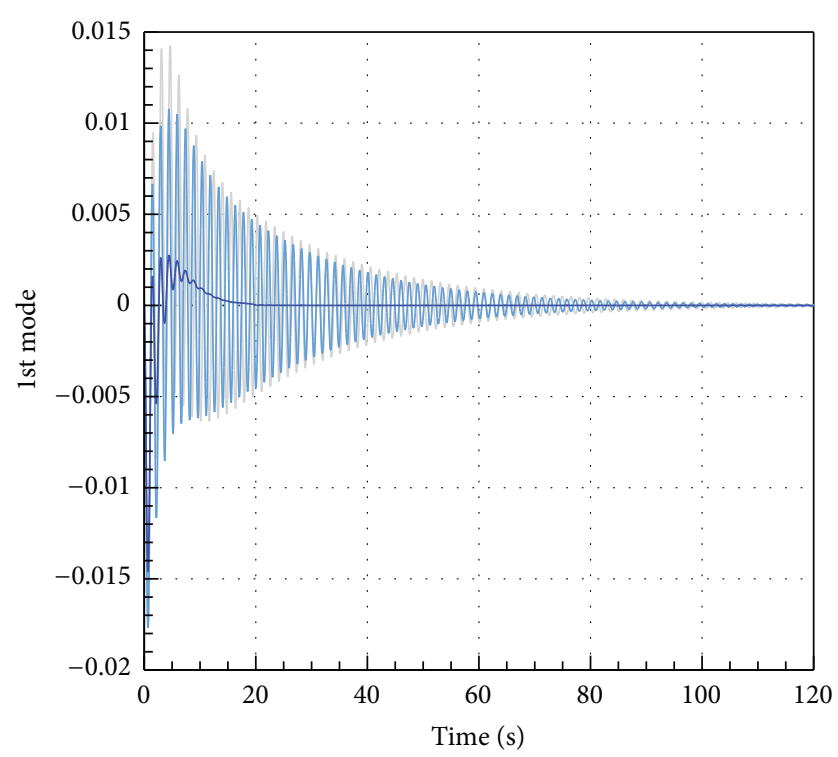

Without PZT action-no SPT usage

Without PZT action using SPT

With PZT action using SPT

FIGURE 7: Time history of 1st vibrational mode.

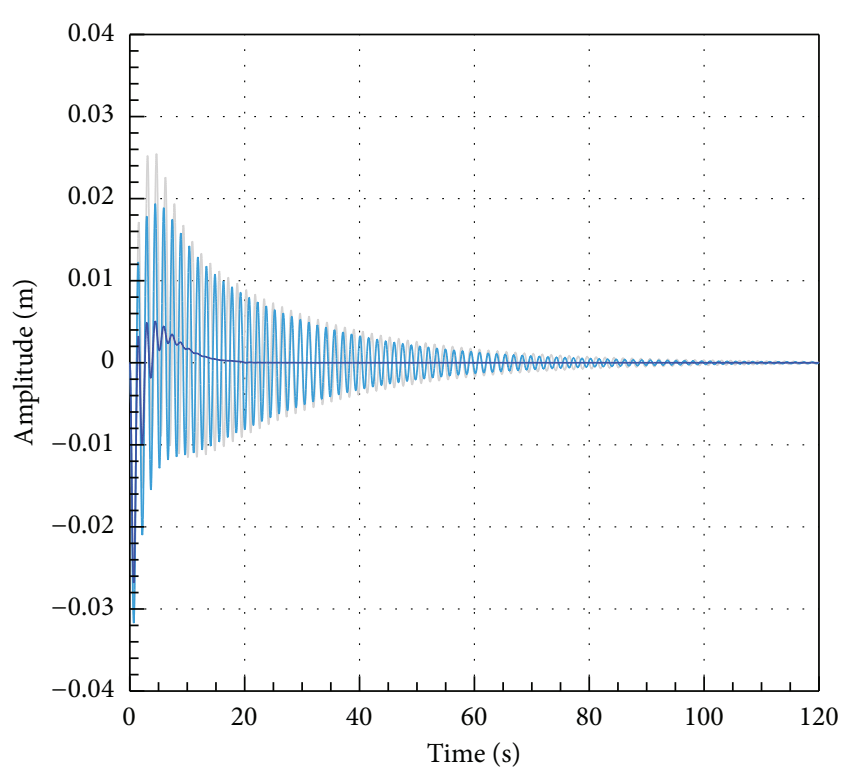

Without PZT action-no SPT action

Without PZT action using SPT

With PZT action using SPT

FIGURE 8: Tip deflection response of the appendage.

$$
\begin{gathered}
C_{R R}^{32}=0 \\
C_{R R}^{31}=-\omega_{x} \sum_{i=1}^{2}\left\{{ }^{i} \Upsilon_{y x}^{T}{ }^{i} \underline{\mathbf{q}}_{k}+{ }^{i} a_{x}{ }^{i} a_{y}+{ }^{i} a_{x}{ }^{i} \Upsilon_{y}^{T}{ }^{i} \underline{\mathbf{q}}_{k}\right. \\
\left.+{ }^{i} a_{y}\left(\rho_{b} \frac{L_{b}^{2}}{2}+\sum_{j=1}^{N_{j}}{ }^{j} \rho_{P} \frac{{ }^{j} L_{P}^{2}}{2}\right)\right\}
\end{gathered}
$$

$$
\begin{aligned}
& -\omega_{y}\left\{\sum_{i=1}^{2}\{\underbrace{i} \mathbf{q}_{k}^{T}{ }^{i} \Upsilon_{y y}^{T}{ }^{i} \underline{q}_{k}\right. \\
& +{ }^{i} a_{y}^{2}\left(\rho_{b}+\sum_{j=1}^{N_{j}}{ }^{j} \rho_{P}\right)+2 a_{y} \\
& \left.\times\left(\rho_{b}+\sum_{j=1}^{N_{j}} \rho_{P}\right){ }^{i} \Upsilon_{y}^{T}{ }^{i} \underline{\mathbf{q}}_{k}\right\} \\
& \left.+\left(I_{Y Y}-I_{X X}\right)\right\} \text {, } \\
& C_{R}^{32}=-\omega_{y} \sum_{i=1}^{2}\left\{{ }^{i} \Upsilon_{y x}^{T} \quad \underline{q}_{k} \mathbf{q}_{k}+{ }^{i} a_{x}{ }^{i} a_{y}+{ }^{i} a_{x}{ }^{i} \Upsilon_{y}^{T}{ }^{i} \underline{q}_{k}\right. \\
& \left.+{ }^{i} a_{y}\left(\rho_{b} \frac{L_{b}^{2}}{2}+\sum_{j=1}^{N_{j}}{ }^{j} \rho_{P} \frac{{ }^{j} L_{P}^{2}}{2}\right)\right\},
\end{aligned}
$$

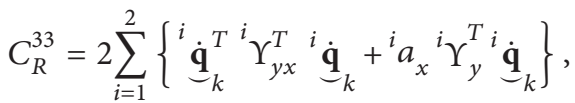

$$
\begin{aligned}
& C_{F R}^{11}=C_{F R}^{21}=C_{F R}^{31}, \\
& C_{F R}^{11}=-\omega_{x}\left\{\left[\begin{array}{cc}
{ }^{1} \Upsilon_{y y} & \mathbf{0} \\
\mathbf{0} & { }^{2} \Upsilon_{y y}
\end{array}\right]^{i} \underline{\mathbf{q}}_{k}\right. \\
& \left.+\left[\begin{array}{cc}
{ }^{1} \Upsilon_{y} & \mathbf{0} \\
\mathbf{0} & { }^{2} \Upsilon_{y}
\end{array}\right]\left[\begin{array}{ll}
{ }^{1} a_{y} & { }^{2} a_{y}
\end{array}\right]^{T}\right\}
\end{aligned}
$$




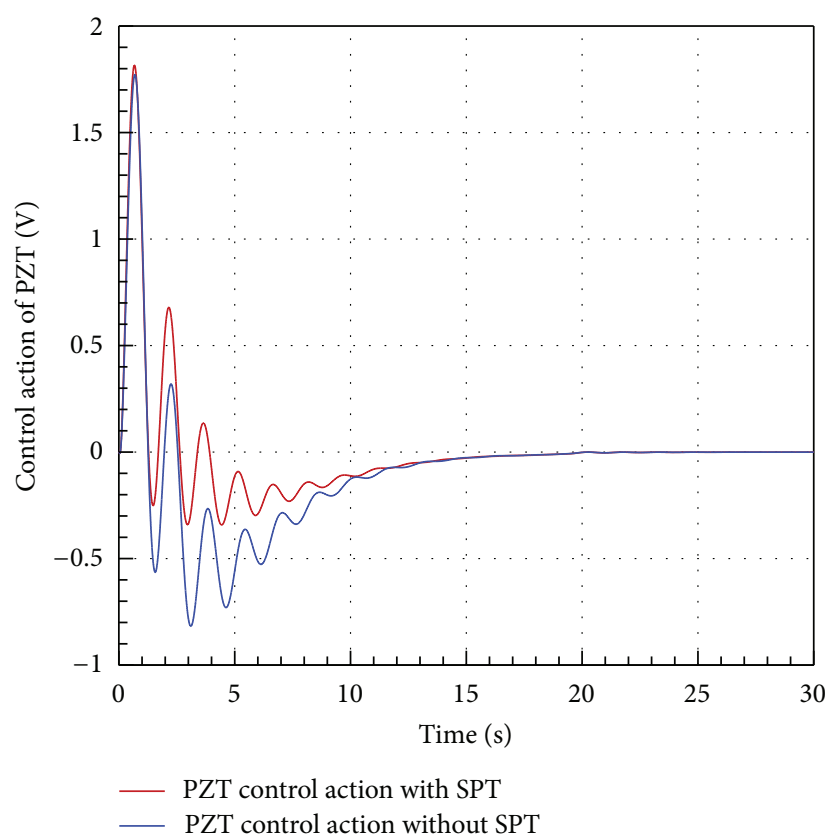

FIgURE 9: Time history of PZT actuation voltage.

$$
\begin{aligned}
& +\omega_{y}\left\{\left[\begin{array}{c}
{ }^{1} \Upsilon_{x y} \\
{ }^{2} \Upsilon_{x y}
\end{array}\right]\left[\begin{array}{cc}
{ }^{1} \Upsilon_{y} & \mathbf{0} \\
\mathbf{0} & { }^{2} \Upsilon_{y}
\end{array}\right]\left[\begin{array}{lll}
{ }^{1} a_{x} & & \\
& & \\
& & \\
x
\end{array}\right]^{T}\right\}, \\
& C_{F R}^{12}=C_{F R}^{22}=C_{F R}^{32}=0, \\
& C_{F R}^{13}=C_{F R}^{23} \\
& =C_{F R}^{33}=-\omega_{z}\{\left[\begin{array}{cc}
{ }^{1} \Upsilon_{y y} & \mathbf{0} \\
\mathbf{0} & { }^{2} \Upsilon_{y y}
\end{array}\right]{ }^{i} \underbrace{}_{k} \\
& \left.+\left[\begin{array}{cc}
{ }^{1} \Upsilon_{y} & \mathbf{0} \\
\mathbf{0} & { }^{2} \Upsilon_{y}
\end{array}\right]\left[\begin{array}{ll}
{ }^{1} a_{y} & { }^{2} a_{y}
\end{array}\right]^{T}\right\} \\
& \mathbf{C}_{R F}=\mathbf{0}, \\
& \mathbf{K}_{F F}=\left[\begin{array}{cc}
1 & 0 \\
\mathbf{0} & { }^{2} \wp
\end{array}\right]{ }^{i} \underbrace{}_{k} \\
& { }^{i} \Upsilon_{y y}=\left(\int_{0}^{L_{b}} \rho_{b}^{i} \psi(x)^{i} \psi^{T}(x) d x\right. \\
& \left.+\sum_{j=1}^{N_{j}} \int_{x_{i}}^{x_{i}+L_{p i}}{ }^{j} \rho_{P}{ }^{i} \psi(x)^{i} \psi^{T}(x) d x\right) \\
& { }^{i} \Upsilon_{y}=\left(\int_{0}^{L_{b}} \rho_{b}{ }^{i} \psi(x) d x+\sum_{j=1}^{N_{j}} \int_{x_{i}}^{x_{i}+L_{p i}}{ }^{j} \rho_{P}{ }^{i} \psi(x) d x\right) \\
& { }^{i} \Upsilon_{y x}=\left(\int_{0}^{L_{b}} \rho_{b}^{i} \psi(x) \cdot x d x\right.
\end{aligned}
$$

$$
\begin{gathered}
\left.+\sum_{j=1}^{N_{j}} \int_{x_{i}}^{x_{i}+L_{p i}}{ }^{j} \rho_{P}{ }^{i} \psi(x) \cdot x d x\right) \\
{ }^{i} \wp=\int_{0}^{L_{b}} E_{b}^{i} I_{b}^{i}\left(\frac{\partial^{2} \psi^{i}(x)}{\partial x^{2}}\right)^{2} d x+\sum_{j=1}^{n_{j}} E_{P}^{i}\left({ }^{j} \omega_{p}^{i}{ }^{j} h_{p}^{i}\right) \\
\times\left({ }^{j} y^{i^{2}}+{ }^{j} y^{i} h_{p} h^{i}+\frac{{ }^{j} h_{p}{ }^{2}}{3}\right) \\
\times \int_{x_{i}}^{x_{i}+L_{P i}}\left(\frac{\partial^{2} \psi^{i}(x)}{\partial x^{2}}\right)^{2} d x .
\end{gathered}
$$

\section{Conflict of Interests}

The authors declare that there is no conflict of interests regarding the publishing of this paper.

\section{References}

[1] D. C. Hyland, J. L. Junkins, and R. W. Longman, "Active control technology for large space structures," Journal of Guidance, Control, and Dynamics, vol. 16, no. 5, pp. 801-821, 1993.

[2] T. Singh and S. R. Vadali, "Input-shaped control of threedimensional maneuvers of flexible spacecraft," Journal of Guidance, Control, and Dynamics, vol. 16, no. 6, pp. 1061-1068, 1993.

[3] Q. Liu and B. Wie, "Robust time-optimal control of uncertain flexible spacecraft," Journal of Guidance, Control, and Dynamics, vol. 15, no. 3, pp. 597-604, 1992. 
[4] S. Vadali, "Feedback control of flexible spacecraft large angle maneuvers using the Liapunov theory," in Proceedings of the IEEE American Control Conference, Piscataway, NJ, USA, 1984.

[5] S. Vadali, J. Junkins, and R. Byers, "Near-minimum time, closed-loop slewing of flexible spacecraft," Journal of Guidance, Control, and Dynamics, vol. 13, no. 1, pp. 57-65, 1990.

[6] S. V. Drakunov and V. Utkin, "Sliding mode control in dynamic systems," International Journal of Control, vol. 55, no. 4, pp. 1029-1037, 1992.

[7] J. Y. Hung, W. Gao, and J. C. Hung, "Variable structure control. A survey," IEEE Transactions on Industrial Electronics, vol. 40, no. 1, pp. 2-22, 1993.

[8] J. D. Bošković, S.-M. Li, and R. K. Mehra, "Robust tracking control design for spacecraft under control input saturation," Journal of Guidance, Control, and Dynamics, vol. 27, no. 4, pp. 627-633, 2004.

[9] J. L. Crassidis and F. L. Markley, "Sliding mode control using modified Rodrigues parameters," Journal of Guidance, Control, and Dynamics, vol. 19, no. 6, pp. 1381-1383, 1996.

[10] Q. Hu, "Variable structure maneuvering control with timevarying sliding surface and active vibration damping of flexible spacecraft with input saturation," Acta Astronautica, vol. 64, no. 11-12, pp. 1085-1108, 2009.

[11] Q. Hu, "Sliding mode attitude control with $L_{2}$-gain performance and vibration reduction of flexible spacecraft with actuator dynamics," Acta Astronautica, vol. 67, no. 5-6, pp. 572-583, 2010.

[12] Q.-L. Hu, Z. Wang, and H. Gao, "Sliding mode and shaped input vibration control of flexible systems," IEEE Transactions on Aerospace and Electronic Systems, vol. 44, no. 2, pp. 503-519, 2008.

[13] J. Kim, J. Kim, and J. L. Crassidis, "Disturbance accommodating sliding mode controller for spacecraft attitude maneuvers," Advances in the Astronautical Sciences, vol. 100, pp. 141-154, 1998.

[14] S.-C. Lo and Y.-P. Chen, "Smooth sliding-mode control for spacecraft attitude tracking maneuvers," Journal of Guidance, Control, and Dynamics, vol. 18, no. 6, pp. 1345-1349, 1995.

[15] Q. Hu and G. Ma, "Vibration suppression of flexible spacecraft during attitude maneuvers," Journal of Guidance, Control, and Dynamics, vol. 28, no. 2, pp. 377-380, 2005.

[16] M. Azadi, S. A. Fazelzadeh, M. Eghtesad, and E. Azadi, "Vibration suppression and adaptive-robust control of a smart flexible satellite with three axes maneuvering," Acta Astronautica, vol. 69, no. 5-6, pp. 307-322, 2011.

[17] M. D. Shuster, "Survey of attitude representations," Journal of the Astronautical Sciences, vol. 41, no. 4, pp. 439-517, 1993.

[18] B. Siciliano and W. J. Book, "A singular perturbation approach to control of lightweight flexible manipulators," The International Journal of Robotics Research, vol. 7, no. 4, pp. 79-90, 1988.

[19] E. Mirzaee, M. Eghtesad, and S. A. Fazelzadeh, "Maneuver control and active vibration suppression of a two-link flexible arm using a hybrid variable structure/Lyapunov control design," Acta Astronautica, vol. 67, no. 9-10, pp. 1218-1232, 2010.

[20] A. H. Meitzler, H. F. Tiersten, A. W. Warner et al., IEEE Standard on Piezoelectricity, IEEE Ultrasonics, Ferroelectrics, and Frequency Control Society, 1988. 

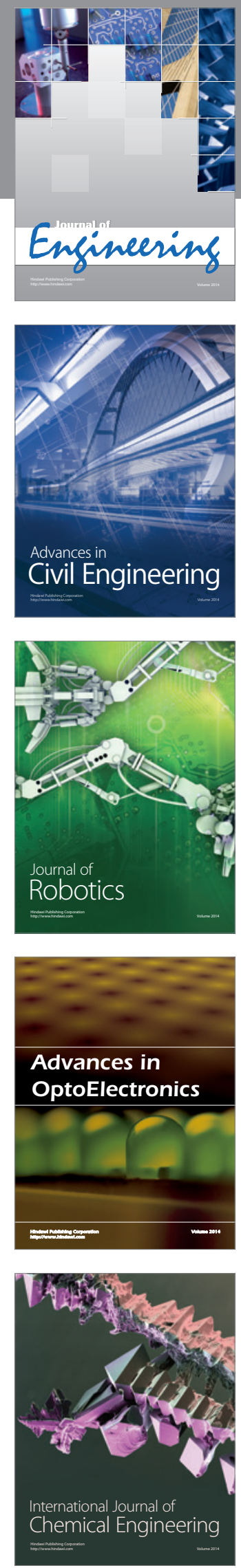

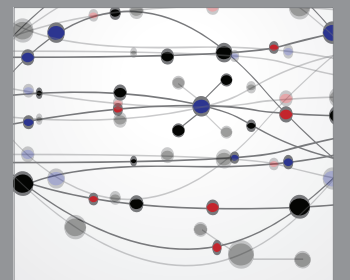

The Scientific World Journal
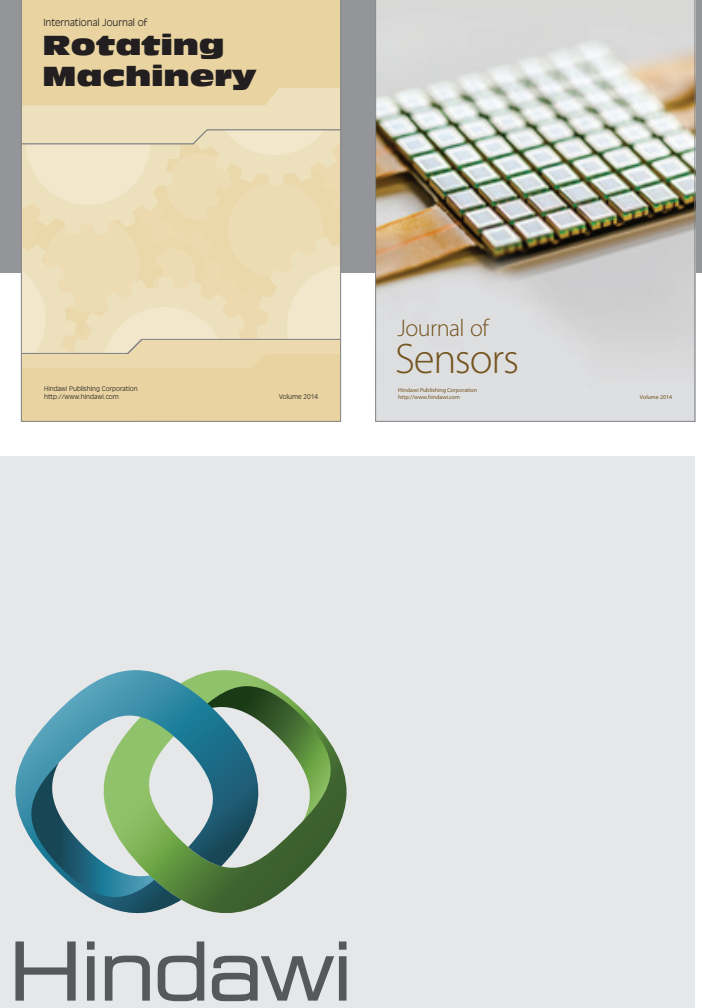

Submit your manuscripts at http://www.hindawi.com
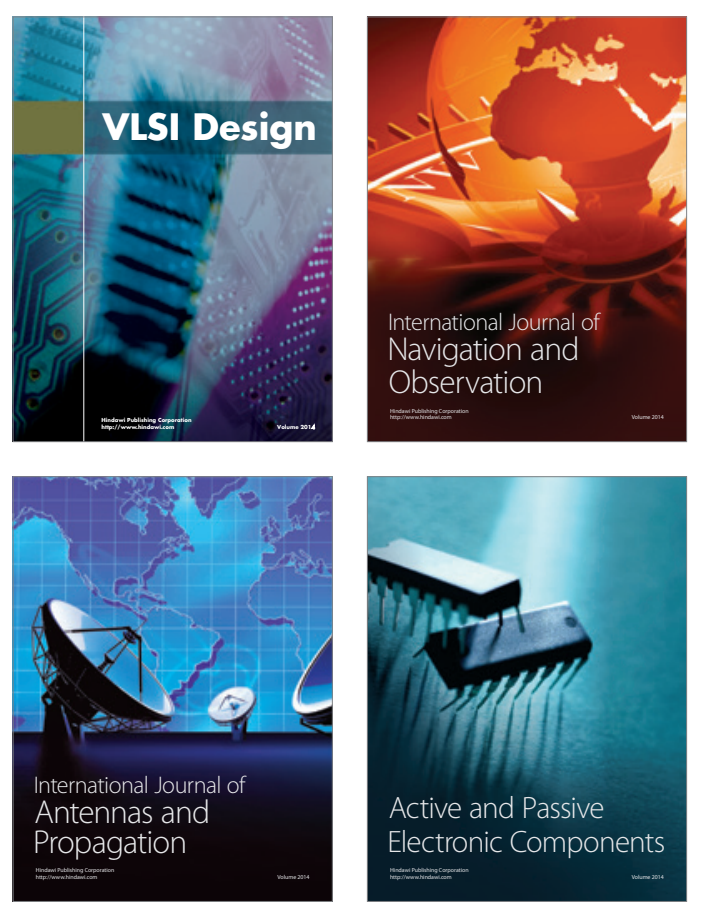
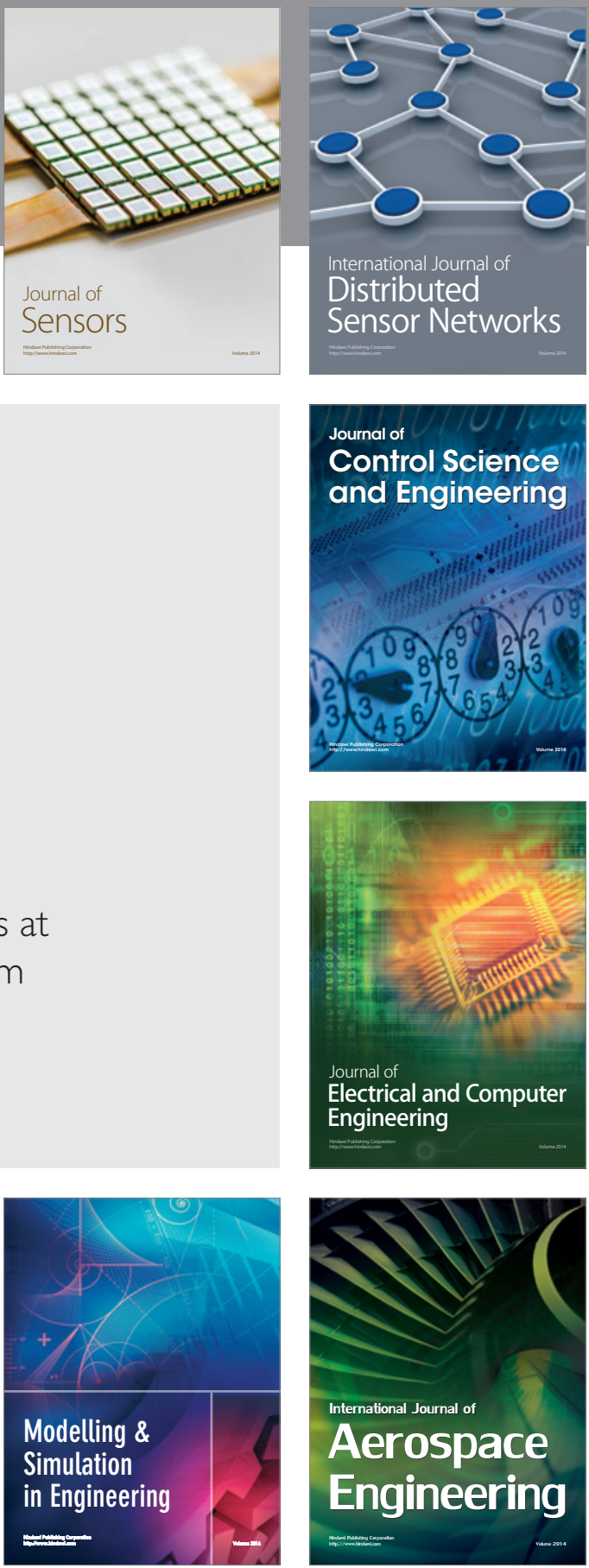

Journal of

Control Science

and Engineering
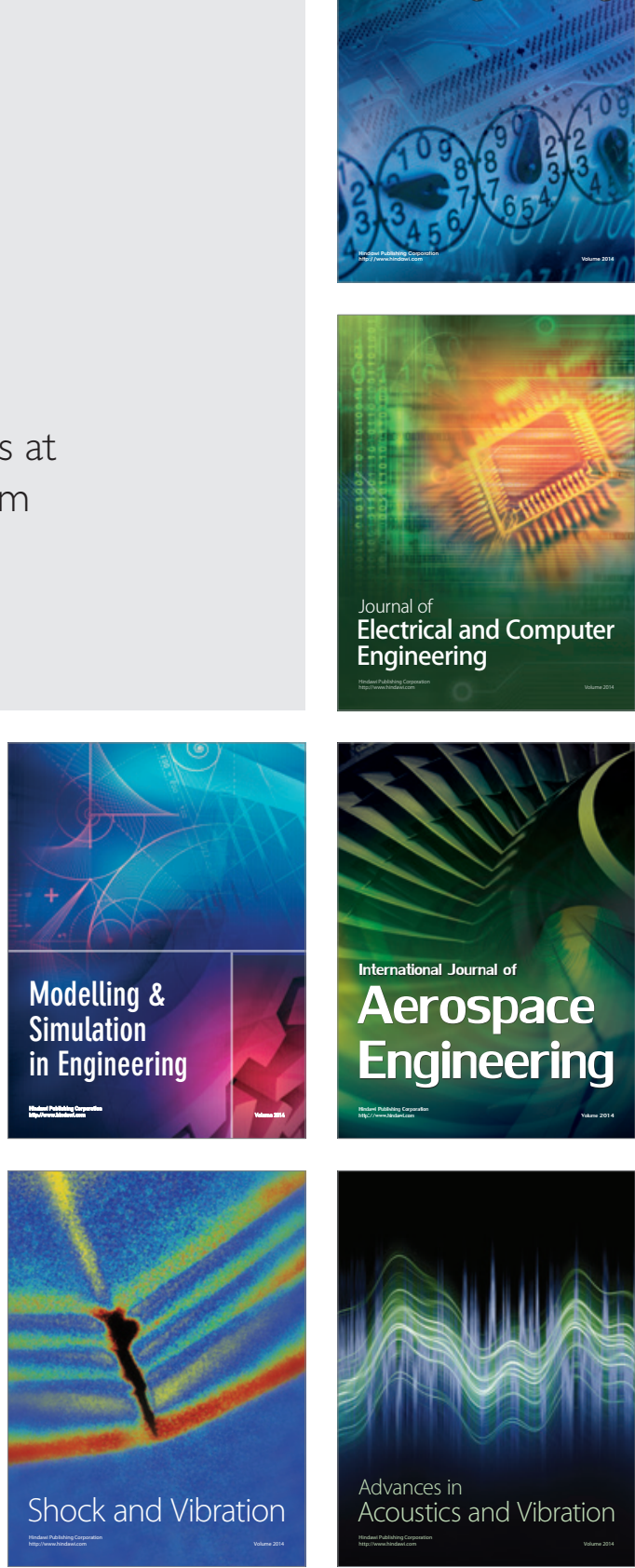